\title{
Preliminary results of the effect of 'Healthy Start', a new food benefit, on the dietary behaviour of women during pregnancy and post partum in Sheffield, UK
}

\author{
Fiona Ford, Robert Fraser, Sarah Wademan and Theodora Mouratidou \\ University of Sheffield, Sheffield, UK
}

Evidence from maternal dietary studies in the UK, suggest that dietary quality and quantity during pregnancy and after delivery decreases with income ${ }^{(1,2)}$. In November 2006 the long-standing Welfare Food Scheme (WFS) was replaced by 'Healthy Start' (HS), ending the provision of volume-based vouchers for liquid cow's milk or infant formula and providing instead monetary vouchers for a broader range of foods including fresh fruit and vegetables. HS is a means-tested benefit aimed primarily at low-income and teenage mothers, together with a greater emphasis on health professionals giving diet, health, lifestyle and parenting advice ${ }^{(3)}$.

The HS project in Sheffield is a cohort study investigating maternal and child nutrition practices in women during pregnancy and post partum and their infants before and after the introduction of the new food benefit scheme. Short-term effects of the introduction of the new scheme on the dietary intakes and eating patterns of two cohorts of women during pregnancy and post partum will be described. Study participants included Caucasian English-speaking women who were eligible for, or recipients of, either WFS milk tokens or 'HS' vouchers. Two non-random samples of women during pregnancy and post partum were recruited in the before-HS and the after-HS study. Dietary intakes of the two cohorts were determined using a validated semi-quantified FFQ at 20 weeks of pregnancy and at 4 weeks after delivery respectively. Mann-Whitney $U$ test was used to compare differences in the percentage of women during pregnancy and post partum meeting the recommended reference nutrient intakes (RNI) ${ }^{(4)}$. Analysis of the preliminary results showed a significant difference in dietary behaviour after the introduction of the new food benefit for women both during pregnancy and post partum. Mean intakes of key nutrients such as energy, folate, Fe and $\mathrm{Ca}$ were significantly higher under the HS scheme. Similarly, an increase in the proportion of women meeting the RNI was observed (Tables 1 and 2). This improvement was primarily a result of increased consumption of fruit and vegetables, fruit juice, meat, fish and oat and wholegrain breakfast cereals.

Table 1. RNI of selected nutrients and percentage of pregnant women under the WFS $(n 83)$ and HS $(n$ 87) schemes meeting the recommendations for pregnancy

\begin{tabular}{lcccc}
\hline & RNI non-pregnant (19-50 years) & \% WFS met the RNI & \% HS met the RNI & $P$ \\
\hline $\mathrm{Ca}$ & $700 \mathrm{mg}$ & 52 & 88 & 0.0001 \\
$\mathrm{Fe}$ & $14.8 \mathrm{mg}$ & 63 & 83 & 0.003 \\
Folate & $200 \mu \mathrm{g}$ & $16^{*}$ & $46^{*}$ & 0.001 \\
Vitamin C & $40 \mathrm{mg}$ & $61^{*}$ & $78^{*}$ & 0.017 \\
\hline
\end{tabular}

*Proportion calculated with the added increment of $100 \mu \mathrm{g}$ for folate and $10 \mathrm{mg}$ for vitamin $\mathrm{C}$ during pregnancy.

Table 2. RNI of selected nutrients and percentage of lactating (LWFS, $n$ 24; LHS, $n$ 12) and non-lactating women (NLWFS, $n$ 56; NLHS, $n$ 50) post partum under WFS and HS schemes meeting the recommendations

\begin{tabular}{lccccccc}
\hline & RNI non-pregnant (19-50 years) & $\%$ LWFS* & $\%$ LHS* & $P$ & $\%$ NLWFS & $\%$ NLHS & $P$ \\
\hline $\mathrm{Ca}$ & $700 \mathrm{mg}$ & $13 *$ & $42^{*}$ & 0.056 & 49 & 80 & 0.001 \\
$\mathrm{Fe}$ & $14.8 \mathrm{mg}$ & 0 & 46 & 0.001 & 5.5 & 20 & 0.026 \\
Folate & $200 \mu \mathrm{g}$ & $39 *$ & $58^{*}$ & 0.279 & 62 & 76 & 0.134 \\
Vitamin C & $40 \mathrm{mg}$ & $48^{*}$ & $68^{*}$ & 0.288 & 71 & 94 & 0.002 \\
\hline
\end{tabular}

*Proportion calculated with the added increment of $550 \mathrm{mg}$ for calcium, $60 \mu \mathrm{g}$ for folate and $30 \mathrm{mg}$ for vitamin $\mathrm{C}$ for lactation.

1. Mouratidou T, Ford F \& Fraser RB (2006) Br J Nutr 96, 929-935.

2. Rogers I, Emmett P, Baker D, Golding J \& the ALSPAC Study Team (1998) Eur J Clin Nutr 52, 251-260.

3. Department of Health (2002) Healthy Start. Proposals for Reform of the Welfare Food Scheme. London: Department of Health.

4. Department of Health (1991) Dietary Reference Values for Food Energy and Nutrients for the United Kingdom. London: H. M. Stationery Office. 